\title{
Examining the Effects of Privacy-Aware Blended Learning Scenarios in Executive Training for Policymakers and Government Officials
}

\author{
Maria Gaci ${ }^{1}$, Juan Carlos Farah ${ }^{1}$, Isabelle Vonèche Cardia ${ }^{1}$, Geneviève \\ Féraud $^{2}$, and Denis Gillet ${ }^{1}$ \\ 1 École Polytechnique Fédérale de Lausanne, Lausanne, Switzerland \\ maria.gaci@epfl.ch \\ 2 United Nations Conference on Trade and Development, Geneva, Switzerland
}

\begin{abstract}
Enabling blended learning scenarios that foster participation and interaction is an important consideration in the design of executive training programs. To investigate this design process, our study focuses on two aspects that are present in such scenarios, namely (1) participant engagement and (2) privacy expectations. This paper presents the pilot deployment of a digital tool within a blended learning scenario implemented by UNCTAD for an executive training program aimed at policymakers and government officials. Our main contribution is a detailed analysis of insights gathered through an observational study with participants from Latin America and the Caribbean. By interpolating data from digital logs, questionnaires, observations, and interviews, our results show that the two aspects investigated play a significant role in fostering interaction among participants. As an outcome of this study, we formulate two hypotheses regarding the design of privacy-preserving blended learning scenarios for validation in future work.
\end{abstract}

Keywords: Blended Learning, Digital Intervention, Privacy, Digitalization, Executive Training

\section{Introduction}

Science, technology, and innovation (STI) are expected to play a major role in achieving the Sustainable Development Goals defined by the United Nations, particularly Goal 8 ("Decent Work and Economic Growth"), Goal 9 ("Industry, Innovation, and Infrastructure") and Goal 17 ("Strengthen the Means of Implementation and Revitalize the Global Partnership for Sustainable Development"). Nonetheless, gaps persist in terms of STI adoption in several regions of the world, especially developing and in transition regions [8].

To provide developing and in transition regions with access to the benefits of STI, the United Nations Conference for Trade and Development (UNCTAD), which is an intergovernmental body of 195 member states established by the United Nations General Assembly, has been promoting technologies that help transform economies [15]. Digitalization, however, requires knowledge transfer 
and digital capacity building, in addition to the adoption of technology. To address this, UNCTAD delivers e-learning programs to train individuals within specific subjects [13], as well as capacity building programs for policymakers and government officials in the framework of a course on Key Issues on the International Economic Agenda, also known as "Paragraph 166" (P166) [14]. The course is organized for developing regions and economies in transition, and provides beneficiaries with state-of-the-art economic and policy knowledge based on UNCTAD research.

Since 2016, UNCTAD and the Swiss Federal Institute of Technology in Lausanne (EPFL) are collaborating to investigate alternative blended learning scenarios that rely on innovative digital tools. Blended learning has been defined by many authors $[2,11]$ and in the context of the present study, refers to the mix of face-to-face and digital activities (e.g., discussions, quizzes) to enhance participation and enrich interaction among participants [3].

Often, a digital tool that acts as a computer-mediated communication channel needs to be deployed in a blended learning scenario [6]. As the introduction of technology may give rise to potential privacy risks and pose important ethical questions, these risks should be considered and appropriately managed [15]. Thus, SpeakUp [12], a digital communication tool ensuring the privacy of participants, was selected for this study.

This paper presents an observational study conducted by UNCTAD and EPFL to investigate the impact of the introduction of a digital tool in the framework of an executive regional course. To frame our study, two potential aspects that might affect participation during training were identified: (i) participant engagement, and (ii) privacy expectations. We present the results of our quantitative and qualitative analyses conducted on data gathered from digital logs, questionnaires, observations, and interviews. Our results guide the formulation of two hypotheses concerning the design of privacy-preserving blended learning scenarios, which we propose to be validated in future work.

\section{Motivation}

Blended learning has been demonstrated to foster interaction between participants [16] and previous studies have shown that social interaction is conditio sine qua non for learning [5]. According to [9], interactions must not be taken for granted, but intentionally designed into the instruction to occur and be meaningful. However, to the best of our knowledge, no studies have been conducted on proposing strategies for increasing interactions (and, as a result, learning) in training programs for policymakers and government officials, hereafter referred to as participants. Thus, this unique collaboration aims to propose strategies that foster face-to-face and digital interactions in such programs.

In recent years, several studies have been conducted to identify aspects that need to be considered when designing digital and/or blended learning scenarios in executive education for Master in Business Administration students $[4,10$, 17]. According to [17], asynchronous communication in online platforms should 
be synchronized with synchronous face-to-face communication to increase participant engagement. Studies conducted by [10] suggest that the dichotomy of humility and hubris should be managed in blended learning scenarios to deliver effective executive education. Performance expectancy, effort expectancy, and hedonic motivation have further proved to be the best predictors of the intention to use blended learning in executive training [4].

In the design of blended learning scenarios for policymakers and government officials, one should consider that participants are representing their respective governments and can be held accountable for their actions and interventions [1]. This translates to specific challenges and requires special consideration. Anonymization due to privacy measures might need to be balanced with the expectation of recognition due to networking goals. Furthermore, the fact that training is contained to three-week periods might help mitigate the risk of dealing with potentially sensitive content by imposing temporal limits on data retention, but might hinder long-term networking possibilities by prematurely closing communication channels for the sake of risk management. Hence, anonymous tools offering different data retention options were introduced in the present executive training program.

Taking into account the trends highlighted in the literature, as well as the specific nature of our participants - as presented above - the design process for the learning scenarios addressed in this paper focuses on two main aspects: Participant Engagement (A1) and Privacy Expectations (A2). Concentrating on these two aspects allows us to formulate hypotheses regarding the factors that could contribute to increased face-to-face and digital interactions among participants. Testing these hypotheses will then provide a baseline for implementing and validating effective blended learning scenarios for executive training aimed at participants of future studies.

Previous studies have been conducted between UNCTAD and EPFL on implementing blended learning scenarios for capacity building for policymakers and government officials [7]. Those studies were concerned exclusively with analyzing data collected from logs generated by the digital tools. However, due to the small sample of participants involved in those previous scenarios, quantitative analyses performed on the logs proved to be insufficient to draw general conclusions. Although the digital logs provided insights into the activity of participants in the digital channel, there was a lack of data regarding face-to-face interaction. To tackle the problems encountered during our previous studies, this paper presents an observational study interpolating different sources of data, comprising digital logs, questionnaires, observations, and interviews.

\section{Methods}

In this section, we first describe the scenario in which the observational study took place. We then introduce SpeakUp. Finally, we outline the different sources from which we were able to gather our data. 


\section{$3.1 \quad$ Use Case}

Our study was conducted in Medellin, Colombia, in July 2019 during the P166 regional course for economies in Latin America and the Caribbean [8]. The study lasted three days and was organized as a part of Module I of the training program, titled "Development, Development Policies, and the Role of International Trade and Finance in a Globalized World". A total of 35 individuals were present in the module: 26 participants ( 11 female \& 15 male), 3 instructors ( 1 female \& 2 male), 3 experts ( 2 female \& 1 male) and 3 researchers ( 1 female \& 2 male).

The module was taught by three different instructors. Sessions were organized with the instructors prior to the beginning of the module to introduce them to SpeakUp and discuss the digital intervention strategy. Each session was held privately and face-to-face with each instructor, and lasted for approximately one hour. The research team from EPFL and one of the experts from UNCTAD were present at each session.

Participants were introduced to SpeakUp through a one-hour briefing session after the opening ceremony. During the briefing, a 20-minute presentation was held, describing the objectives of the observational study and briefly explaining SpeakUp's main features. Participants then had the opportunity to practice using the digital tool and ask questions.

Throughout the training, participants were seated in a horseshoe/modified$\mathrm{U}$ arrangement composed of two rows in a conference room. All participants had at least one digital device (mobile phone, laptop, and/or tablet) to connect to SpeakUp. The official languages of the training material were English and Spanish. During training, researchers from EPFL were present to observe the digital interaction and provide technical assistance.

\subsection{Digital Tool}

Virtual interaction during training was enabled through the use of SpeakUp, a digital chatroom designed to foster social interaction and collaboration of participants co-located in physical spaces. In a typical scenario, the instructor creates a chatroom before the start of the presentation, and participants can join (without registration or login), post messages, vote on existing messages using the like/dislike button, and answer multiple-choice questions (Fig. 1). The absence of authentication enables participants to start using the tool with little friction while maximizing their anonymity.

To manage the privacy of the content generated in the chatroom, SpeakUp offers chatroom creators the option of configuring two features: Nicknames and Here $\&$ Now. The former defines the anonymity of participants and the latter the persistence of the data generated during the sessions. Thus, privacy in the current context is defined in terms of these two features. More specifically, it is defined by the requirement of nicknames (or lack thereof) and the duration for which the chatroom will remain accessible, respectively. The two options available regarding anonymity are anonymous and nicknames, while the two options for data retention are temporary (24 hours) and permanent (or until 



Fig. 1. Two screenshots of SpeakUp. Left: Instructors can create new chatrooms. Right: Participants can join, post messages, vote, and answer multiple-choice questions.

manually deleted by the chatroom administrator). The most privacy-aware case is implied to be the anonymous and temporary chatroom, and the least privacyaware one is implied to be the pseudonymous and permanent chatroom. In the context of this study, only temporary chatrooms were assessed.

The configurations of the chatrooms created during the training were the following:

- Anonymous \& Temporary (Days 1 and 3)

- Pseudonymous (Nicknames) \& Temporary (Day 2)

\subsection{Data Collection}

A mixed-methods approach was employed to gather data, combining quantitative and qualitative methods using four techniques: log file analysis obtained from SpeakUp (T1), questionnaires (T2), observations (T3), and interviews (T4). Data gathered was interpolated to enrich the evaluation process and provide insights into the digital interaction (T1), the face-to-face interaction (T3), and both (T2, T4). Table 1 provides a detailed chronological overview of the data collected during the study.

Log files were extracted automatically using SpeakUp's administrator privileges. The activity of the participants and the instructors in the chatroom was exported at the end of each session in the form of a comma-separated (CSV) file. Each log file contained information regarding the number of messages written in 
Table 1. Chronological Overview of the Activities

\begin{tabular}{lccc}
\hline Activity & \# Individuals & Techniques & Aspects \\
\hline Briefing Session w/ Participants & 26 & - & $\mathrm{A} 2$ \\
Pre-Questionnaire & 26 & $\mathrm{~T} 2$ & $\mathrm{~A} 2$ \\
Session 1 & 26 & $\mathrm{~T} 1, \mathrm{~T} 3$ & $\mathrm{~A} 1$ \\
Interviews with Participants & 2 & $\mathrm{~T} 4$ & $\mathrm{~A} 1, \mathrm{~A} 2$ \\
Session 2 & 26 & $\mathrm{~T} 1, \mathrm{~T} 3$ & $\mathrm{~A} 1$ \\
Session 3 & 26 & $\mathrm{~T} 1, \mathrm{~T} 3$ & $\mathrm{~A} 1$ \\
Interviews with Participants & 3 & $\mathrm{~T} 4$ & $\mathrm{~A} 1, \mathrm{~A} 2$ \\
Post-Questionnaire & 26 & $\mathrm{~T} 2$ & $\mathrm{~A} 2$ \\
\hline
\end{tabular}

the online chatroom along with the content, timestamp, nickname (if applicable), likes, and comments per message.

Non-participant observations were conducted during the three-day training by one of the researchers of the EPFL team to record the face-to-face interactions. As participants were sitting on pre-assigned seats throughout the duration of the training, a unique random code was allocated to each of them. The observer would then note down in a confidential notebook the code of the participant interacting at each moment, along with the type of interaction (e.g., "asking a question", "providing an answer", "clarifying").

Two separate questionnaires were distributed to participants at the beginning (pre) and at the end (post) of the module $(n=26)$. Five-level Likert questions following a scale from "Strongly Disagree" to "Strongly Agree" were included to evaluate how participants perceived privacy, as well as their intended (pre) and actual (post) behavior in the chatrooms. Open-ended questions were included at the end of the questionnaires for the participants to share their expectations and to gather comments, feedback, and queries.

Finally, face-to-face unstructured interviews were organized individually with five of the participants. Each interview lasted between 20 and 30 minutes. A narrative analysis approach was selected to review the individual responses of each participant regarding the effective design of privacy-aware blended learning scenarios to highlight critical points and find common patterns.

\section{Results}

The various qualitative and quantitative sources detailed in Section 3 were analyzed and triangulated to address the two aspects presented in Section 2. Onesample Wilcoxon signed-rank tests were performed on the data obtained through the two questionnaires to test for significant divergence from the expected median Likert score of three. The results of the analysis are presented below, organized by aspect. 


\subsection{Participant Engagement (A1)}

Face-to-face interaction was analyzed for each of the three sessions. During each session, 18, 9 and 18 participants, respectively, asked/provided face-to-face questions/answers (Table 2). In total, five participants asked face-to-face questions in all sessions, 12 participants in any two of the three sessions, 10 participants in only one session, and two participants did not ask any face-to-face questions at all.

Digital interaction was also analyzed for each of the three sessions (Table 2). If the number of participants attending the training $(n=26)$ is compared to the number of registered users on SpeakUp - 50, 38, and 25 for each session, respectively - it may be inferred that almost everyone used the digital channel, even though the utilization was not compulsory. The higher number of registered users in the chatroom is a result of participants utilizing multiple devices during the training. This was evident when inspecting the number of nicknames during the second session, when despite the fact that 38 users were registered, only 26 unique nicknames were recorded. Overall, $46 \%, 39 \%$, and $28 \%$ of the participants who joined the digital room in each session posted at least one message, while $22 \%, 37 \%$, and $20 \%$ of the participants in each session voted at least once.

Table 2. Face-to-Face vs Digital Interaction

\begin{tabular}{llccc}
\hline Interaction & Session 1 Session 2 Session 3 \\
\hline \multirow{5}{*}{ Face-to-Face } & Interaction Type & 4 & 2 & 5 \\
& Question Posed by Instructor & 7 & 8 & 17 \\
& Question Posed by Participant & 27 & 4 & 19 \\
& Comment / Opinion & 2 & 0 & 10 \\
\hline \multirow{5}{*}{ Digital } & Clarification & 3 & 0 & 1 \\
& Poll Created by Instructor & 2 & 37 & 5 \\
& Vote & 28 & 6 & 6 \\
& Question Posed by Participant & 15 & 5 & 1 \\
\hline
\end{tabular}

According to three participants, questions that were not urgent and required the opinion of a broader audience were typically posted in the digital chatroom, as opposed to questions that required immediate clarification from the instructor. We observed that $22 \%$ of the questions were asked to clarify content presented in the slides, such as several graphs and equations. Participants expressed a positive attitude towards the use of digital tools to get a diverse range of opinions from peers, especially as it did not interrupt the flow of the presentation and they could continue the discussion online after the training.

Furthermore, we observed that the tool was highly utilized by participants speaking a different native language than the one used during the presentation. 
Participants would post messages in the digital chatroom in either of the two languages and other participants would volunteer to translate them into the other language. In total, 14, 5, and 3 of the messages posted in the digital chatroom were translated for each session, respectively.

Contrary to the results of previous studies conducted in higher education settings [12], spamming was neither an issue in the fully anonymous scenarios nor the pseudonymous scenario. Zero spam messages were recorded in the chatrooms and participants pointed out that spamming was not relevant in the current context as it was a professional setting predominantly focused on learning.

\subsection{Privacy Expectations (A2)}

Several questions were included in the pre- and post-questionnaires to gain insight into the participants' perception of privacy. In the pre-questionnaire, participants stated that ensuring privacy would result in higher participation in the digital chatrooms (mean Likert score $\bar{x}=4.15, S D=0.95, Z=29.0, p<0.001$ ). Participants also considered full anonymity to be an important way of ensuring privacy $(\bar{x}=3.85, S D=1.03, Z=18.0, p<0.001)$.

In the pre-questionnaire, participants expressed that they would participate more if the digital tool was fully anonymous $(\bar{x}=3.52, S D=0.8, Z=13.0$, $p<0.01)$ and if posts were deleted automatically after some time $(\bar{x}=3.37$, $S D=0.97, Z=18.0, p<0.05)$. In the post-questionnaire, participants were asked to reflect on their behavior and identify if anonymity $(\bar{x}=2.76, S D=0.97$, $Z=40.0, p=0.23)$ and ephemerality of data $(\bar{x}=2.71, S D=0.95, Z=31.0$, $p=0.15$ ) affected the rate of digital interaction (Table 3). Participants were also asked if knowing the identity of participants affected the trust on the messages posted in the virtual chatroom and a mean Likert score of $\bar{x}=3.52(S D=0.89$, $Z=21.0, p=0.01)$ was obtained.

During the interviews, participants were indirectly posed questions regarding their behavior in the virtual chatroom. A group of them mentioned that they considered themselves introverts and the availability of a digital channel enabled them to prepare questions in advance, formulate them correctly, and feel less anxious about expressing their opinions. Furthermore, anonymity was considered to be important, as it enabled participants to express themselves without the risk of encountering negative verbal and nonverbal reactions from their peers and instructors. Participants were asked if the quality of the posts on the digital chatroom would be higher if participants posted anonymously and the mean Likert score was $\bar{x}=3.48(S D=0.80, Z=24.0, p<0.01)$.

Three participants mentioned that anonymous digital channels could be extremely useful in scenarios where people can be judged depending on their hierarchical status and position, yet they were not necessary for training. They suggested that knowing the identity of the participants is necessary to build connections, and as policymakers and government officials, they are trained to overcome the fear of public speaking. When participants were asked about the importance of knowing the identity of other participants to build connections, the mean Likert score was $\bar{x}=3.48(S D=0.89, Z=33.0, p<0.05)$. 
Table 3. Questions Repeated in the Pre- and Post-Questionnaires

\begin{tabular}{lcccc}
\hline $\begin{array}{l}\text { On a scale from } 1 \text { (Strongly Disagree) to } 5 \text { (Strongly Agree), } \\
\text { how would you rate the following statements? }\end{array}$ & $\bar{x}$ & \multicolumn{2}{c}{ Post } \\
& $\bar{x}$ & SD \\
\hline I am/was concerned about privacy when posting on SpeakUp. & 3.44 & 1.22 & 2.52 & 1.19 \\
I will/did participate more if I am/was anonymous. & 3.52 & 0.80 & 2.76 & 0.97 \\
I will/did participate more if posts are/were temporary. & 3.37 & 0.97 & 2.70 & 0.95 \\
\hline
\end{tabular}

To better understand the effect of pseudonymity, we performed an analysis of the nicknames selected by the participants during the second session. Before the start of the session, participants were informed about two options when choosing a nickname: (a) use of their real names or initials, so that they would be identifiable, and (b) use of a fictional name, so that they would be unidentifiable. The nicknames selected were as follows. Two participants used their first name, four participants used the initials of their full names and 20 participants selected objects, fictional characters, professional titles, or other unidentifiable names.

In the post-questionnaire, participants were asked to select the chatroom settings that they would prefer for two different use cases: (i) if they were the creators of the chatroom and were going to use the tool as an instructor and (ii) if they were users of the chatroom (Fig. 2). A total of $64 \%$ of participants selected the same settings in both use cases and $68 \%$ of the participants preferred temporary chatrooms either as creators or users. Approximately $52 \%$ preferred the fully anonymous setting as users of the chatroom, but only $40 \%$ preferred the fully anonymous setting as creators of the chatroom. The most preferred combination of settings in both scenarios was "temporary chatroom with pseudonymity". Participants stated that the preferred settings would differ according to the context of each scenario, as sometimes it is of high importance to have access to the content of a meeting (hence, permanent chatrooms were desired) and sometimes it is of high importance to ensure that everyone has participated and shared their opinion (hence, anonymity was not highly favorable).

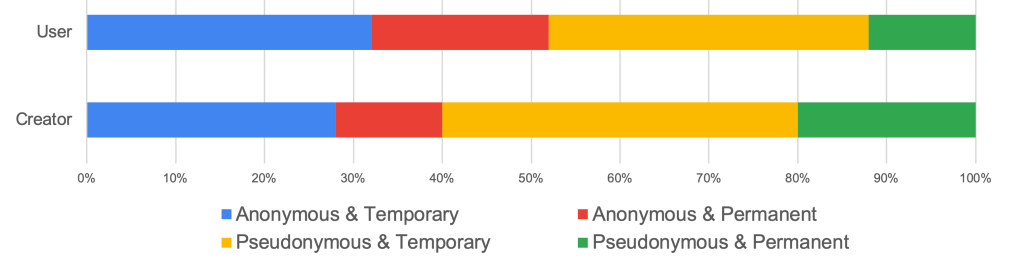

Fig. 2. Difference in preferred chatroom privacy settings when considering the perspective of a chatroom user or a chatroom creator/administrator. 
Before the intervention, participants were neutral about the statement "I will be concerned about privacy when posting on a SpeakUp chatroom" $(\bar{x}=3.44$, $S D=1.22, Z=82.5, p=0.08)$. However, at the end of the module, the mean Likert score dropped to $\bar{x}=2.52(S D=1.19, Z=68.0, p<0.05)$, suggesting that participants were slightly less concerned about their privacy after being exposed to the tool. Nonetheless, despite its privacy-preserving features, participants did not indicate a preference to use SpeakUp over asking face-toface questions $(\bar{x}=3.02, S D=0.98, Z=49.0, p=0.82)$. Feedback from the interviews and observations during the training revealed that privacy remained the participants' main concern (Table 3), but this was mainly attributed to the setup of the training and not the use of SpeakUp, as summarized below.

Firstly, due to the layout of the room, participants sitting in the second row could observe the screen of the participants sitting in the first row. Secondly, participants could deduce who was the author of the message by matching the person typing and the messages posted at a specific time due to the small group size. Thirdly, in the case of pseudonymous chatrooms, participants could read their neighbors' nicknames, as the physical distance between adjacent participants was relatively small. Moreover, as participants knew each other in a professional context, they claimed that they could sometimes identify patterns in the posts due to keywords or due to the structure of the language used. Lastly, nicknames were mentioned to create differences between the participants, as some nicknames were referred to as being more favorable and prompting, thus affecting the rate of comments and likes.

\section{Discussion}

This observational study aimed to investigate two aspects of blended learning scenarios for training for policymakers and government officials in Latin America and the Caribbean: participant engagement (A1) and privacy expectations (A2). By interpolating quantitative and qualitative data gathered from four different sources, we can summarize our findings per aspect as follows.

$A 1$. The addition of a computer-mediated communication channel can encourage participant interaction in bilingual settings. Furthermore, different communication channels can be used to interact with instructors depending on the perceived urgency of a question.

A2. Although enforcing digital privacy is essential, it is not sufficient to encourage user participation in training. Failing to take into account physical privacy in small co-located group settings, as well as the desire of participants to have a digital identity that exposes their participation, could be detrimental to fostering participant interaction.

The combination of the two findings in this paper suggests that a correlation exists between participants' engagement and privacy expectations. Therefore, two hypotheses can be formulated for future investigation. 
- A computer-mediated communication channel that takes into consideration the urgency of a question will result in increased participant interaction in blended learning scenarios for executive training programs. (H1)

- A computer-mediated communication channel that provides privacy options at the interaction level-rather than at the user level-will foster participant engagement in blended learning scenarios for executive training programs. (H2)

Validation of these two hypotheses in future studies will contribute to the ultimate goal of the collaboration between UNCTAD and EPFL. That is, proposing best practices for blended learning scenarios for executive training for policymakers and government officials that encourage both face-to-face and digital interactions.

\section{Conclusion}

This study analyzed the use of a privacy-aware digital tool in a blended learning scenario comprising the three instructors and 26 participants of a UNCTAD regional course in Latin America and the Caribbean. In particular, two aspects faced in such a framework (participant engagement and privacy expectations) were explored. The results of this study suggest that the two factors should be taken into consideration when designing blended learning scenarios for executive training for policymakers and government officials.

Our findings reveal that digital tools could complement face-to-face interaction by providing new means of collecting a wide range of opinions during training. Furthermore, results show that in the current context, privacy ensured by the digital tool was not sufficient to increase digital interactions, as participants realized that their privacy could be compromised by the physical setting of the scenario. Thus, even though the two aspects investigated play an important role in fostering interaction among participants, their correlation could not be determined. Instead, two hypotheses $(\mathrm{H} 1, \mathrm{H} 2)$ were formulated to be addressed in future work.

To conclude, this paper describes the initial experience of deploying a digital tool in executive training for policymakers and government officials. Despite the limitations of a small sample size and a specific use case, our findings have practical implications that could support future research on the impact of novel digital tools in blended learning scenarios aimed at policymakers and government officials.

\section{Acknowledgments}

This research has been co-funded by the European Union's Horizon 2020 research and innovation program in the context of the Next-Lab Innovation Action and the Marie Skłodowska-Curie Action (grant agreement nos. 754354 and 731685). 


\section{References}

1. Adsera, A., Boix, C., Payne, M.: Are you being served? Political accountability and quality of government. The Journal of Law, Economics, and Organization 19(2), 445-490 (2003)

2. Asare, A.O., Yun-Fei, S., Adjei-Budu, K.W.: Adoption of e-learning in higher education: Expansion of UTAUT model. European Academic Research 3(12), 13,23613,259 (2016)

3. Chen, N.S., Wei, C.W., Chen, Y.R., Wang, Y.C.: Bridging the gap between faceto-face and cyber interaction in holistic blended learning environments. Handbook on Information Technologies for Education and Training pp. 239-259 (2008)

4. Dakduk, S., Santalla-Banderali, Z., van der Woude, D.: Acceptance of blended learning in executive education. SAGE Open 8(3) (2018)

5. Erickson, J., Siau, K.: E-ducation. Communications of the ACM 46(9), 134-140 (2003)

6. Everett, D.R., Ahern, T.C.: Computer-mediated communication as a teaching tool: A case study. Journal of Research on Computing in Education 26(3), 336-357 (1994)

7. Féraud, G., Holzer, A., Vonèche Cardia, I., Gillet, D.: ICT adoption in executive training for development: Blending digital and physical communication and awareness channels. In: Proceedings of the Tenth International Conference on Information and Communication Technologies and Development, pp. 1-4 (2019)

8. Hilbert, M.R.: Latin America on its path into the digital age: Where are we? United Nations Publication (2001)

9. Kreijns, K., Kirschner, P.A., Jochems, W.: Identifying the pitfalls for social interaction in computer-supported collaborative learning environments: A review of the research. Computers in Human Behavior 19(3), 335-353 (2003)

10. Lockhart, J., McKee, D., Donnelly, D.: Delivering effective blended learning: Managing the dichotomy of humility and hubris in executive education. Decision Sciences Journal of Innovative Education 15(1), 101-117 (2017)

11. Martín García, A.V., García del Dujo, A., Muñoz Rodríguez, J.M.: Determinant of blended learning adoption in higher education. Adaptation of the UTAUT model. Educación XX1 17(2), 217-240 (2014)

12. Rodríguez-Triana, M.J., Holzer, A., Prieto, L.P., Gillet, D.: Examining the effects of social media in co-located classrooms: A case study based on SpeakUp. In: European Conference on Technology Enhanced Learning, pp. 247-262. Springer (2016)

13. UNCTAD: UNCTAD e-Learning on Trade. URL https://elearningtrade.unctad. org. Accessed: 2020-06-30

14. UNCTAD: UNCTAD Paragraph 166 Course. URL https://p166.unctad.org. Accessed: 2020-06-30

15. UNCTAD: Harnessing frontier technologies for sustainable development. United Nations Publication (2018)

16. Wang, M.: Online collaboration and offline interaction between students using asynchronous tools in blended learning. Australasian Journal of Educational Technology 26(6), 830-846 (2010)

17. Wolverton, C.C.: Utilizing synchronous discussions to create an engaged classroom in online executive education. The International Journal of Management Education 16(2), 239-244 (2018) 